\title{
The Association between Late Third-Trimester Oxytocin Level and Early-Onset Postpartum Depression Symptoms among Jordanian Mothers: A Cross-sectional Study
}

\author{
Hasan Rawashdeh (D, ${ }^{1}$ Zahra Alalwani, ${ }^{2}$ Amer Sindiani, ${ }^{1}$ Rana Alodetalah, ${ }^{3}$ \\ and Mohammad Alqudah $\mathbb{1}^{4}$ \\ ${ }^{1}$ Department of Obstetrics and Gynaecology, Jordan University of Science and Technology (JUST), Post Office Box 3030, \\ Irbid 22110, Jordan \\ ${ }^{2}$ King Abdulla University Hospital (KAUH), Ar-Ramtha, Jordan, Post Office Box: 630001, Irbid, 22110, Jordan \\ ${ }^{3}$ Department of Obstetrics and Gynecology at King Abdulla University Hospital (KAUH), Ar-Ramtha, Post Office Box: 630001, \\ Irbid, 22110, Jordan \\ ${ }^{4}$ Department of Pathology and Microbiology, Jordan University of Science and Technology (JUST), Post Office Box 3030, \\ Irbid 22110, Jordan \\ Correspondence should be addressed to Hasan Rawashdeh; hmrawashdeh@just.edu.jo
}

Received 11 September 2021; Revised 13 December 2021; Accepted 3 February 2022; Published 14 February 2022

Academic Editor: Janusz K. Rybakowski

Copyright (c) 2022 Hasan Rawashdeh et al. This is an open access article distributed under the Creative Commons Attribution License, which permits unrestricted use, distribution, and reproduction in any medium, provided the original work is properly cited.

\begin{abstract}
Purpose. Oxytocin has been suggested to play a vital role in modulating maternal behavior and stress-related disorders. However, the relationship between antenatal oxytocin and postpartum depression is not well established. We aim to investigate the association between serum oxytocin level in the late third-trimester and early-onset postpartum depression symptoms. Materials and Methods. A total of 172 healthy pregnant women participated in this cross-sectional descriptive study. The serum oxytocin level was measured between 34 and 37 weeks. A validated Edinburgh Postnatal Depression Scale (EPDS) was used to assess symptoms of depression four to six weeks postpartum. Participants who scored more than 12 on the EPDS were considered having depressive symptoms. Independent sample $t$-test and Pearson $r$ were used to examine differences in depression scores. The level of significance was set at $\alpha=0.05$. Results. 30.8\% of the participants experienced depressive symptoms. There was no association between EPDS scores and oxytocin level $r(170)=0.10, p=0.23$ . The association also did not exist even among women with a lifetime history of depression $r(43)=-0.13, p=0.37$. Participants with low education, low income, previous history of depression, positive family history of depression, positive family issues, and absent emotional family support have scored significantly higher on EPDS scores than their counterparts. The strongest association was with previous lifetime history of depression $t(170)=-4.40, p<0.001$. Conclusions. Postpartum depression is a major public health problem in Jordan. Late trimester serum oxytocin level has no association with early-onset postpartum depression.
\end{abstract}

\section{Introduction}

Pregnancy is a major life event that is inevitably accompanied by social, psychological, and hormonal changes. In fact, from a psychological point of view, it is considered the most complex event in human experience. Pregnancy can trigger a wide range of emotions from transient mood liability to severe depressive episodes [1].

Traditionally, postpartum psychiatric disorders (PPPD) have been classified into postpartum blues, postpartum psychosis, and postpartum depression. Postpartum depression (PPD) is the most common form and is defined as a 
depressive episode, starting within 6 months after childbirth, that meets the DSM-IV criteria for a major depressive episode, without psychotic features [2].

In Jordan, the incidence of PPD is about $22 \%$, which is comparable to the nearby countries [3-5]. It places PPD as one of the most common and most serious public health problems since it is a major contributor to the $8 \%$ of maternal deaths related to mental health problems [6]. Moreover, infants of PPD mothers are at significant risk of physical, emotional, cognitive, and interpersonal problems in their later lives [1].

PPD is often underdiagnosed and untreated for many reasons related to maternal underreporting of symptoms, poor awareness of the medical care providers, and limited sensitivity of the available screening tools [7]. On the other hand, there is good evidence showing that PPD can be prevented if prophylactic antidepressants were commenced immediately after delivery for the high-risk population [8]. This has urged researchers to explore possible associations between PPD and specific antenatal biomarkers that have the potential to predict and identify PPD objectively before symptoms develop.

Many biomarkers were studied like oxytocin level, leptin level, brain-derived neurotrophic factor (BDNF), and luteinizing hormone: follicular stimulating hormone ratio, but the oxytocin level is the best-studied brain system in humans with vast growing evidence showing its effect on social perception, behavior, and social memory, the amygdala, and stress-related disorders [9-14].

Oxytocin is a peptide hormone synthesized in the supraoptic and paraventricular nuclei of the hypothalamus and released into the bloodstream via the posterior pituitary gland. Due to the prosocial effects of oxytocin along with its antidepressant and anxiolytic properties, we decided to test its association with PPD. In this study, we aim to investigate the association between serum oxytocin concentration in the late third-trimester and postpartum depression in the early postnatal period among Jordanian mothers.

\section{Materials and Methods}

In this cross-sectional descriptive study, the participants were sampled from the antenatal clinics at King Abdulla University Hospital (KAUH) which is the largest tertiary hospital in the north of Jordan. During the regular late antenatal visits, every pregnant woman attending the clinics was interviewed by a designated interviewer where a full description of the study design was delivered. The interviewer was a medical practitioner who collected a brief socioeconomic history and provided instant answers for the candidates' inquiries before completing the written informed consent of participation if they have fulfilled the inclusion criteria and agreed to participate. All candidates were allowed to withdraw from the study at any time.

The sample size was computed utilizing the G* Power software [15]. A power level of 0.80 , an alpha level of 0.05 , and an effect size of 0.5 for the independent $t$-test were used to calculate the sample size. The estimated sample size was 128 based on the previous parameters. The study's final sample size was 172 participants.
2.1. Subjects. The participants were mothers having a singleton pregnancy, between 34 and 37 weeks of gestation, not known to have chronic medical illnesses nor current mental illnesses, not known to have fetal abnormalities whether growth, structural, or chromosomal, and no history of neonatal deaths for the current pregnancy. Both primgravid and multigravida women were included. Gestational age was confirmed by reviewing the first-trimester scan report between 11 and 14 weeks using the crown rump length (CRL) measurement.

Over 16 months' duration, starting in September 2018 and ending by December 2019, 211 participants were admitted to the study. 14 of them declined to provide a blood sample and asked to be withdrawn. 197 blood samples were collected. Follow-up was lost with 24 participants because they provided the wrong contact details or they failed to answer within the specific period. There was one case of early neonatal death after one week of delivery where the entry was removed. The final complete sample was consisted of 172 participants where they completed the consent of participation, provided blood sample for serum oxytocin concentration, and answered our phone call after 4 to 6 weeks of their delivery of a live singleton newborn and completed the Edinburgh Postnatal Depression Scale (EPDS) by the same interviewer. A flowchart of participants is displayed in Figure 1.

EPDS is the most commonly used validated screening tool for postpartum depression [16, 17]. A score of 13 and above is the most widely used score to define probable depression [18]. A validated translation of EPDS into the Arabic language was used to assess postpartum depression symptoms [17]. A total of 10 items, dealing with typical PPD symptoms, were answered on a 4-point scale. Women who scored 13 and above were considered positive for depression.

Ethical approval was gained by the IRB committee (Reference number: 48/116/2016 on 31/05/2018). Demographic and clinical data were collected from the medical records, while socioeconomic data were completed during signing the consent. All participants had completed and signed the informed consent for participation.

2.2. Sample Collection and Storage. All experimental work took place at the research laboratory located at the same hospital. All safety protocols were applied to all procedures. Biohazardous materials were disposed of according to the biosafety guideline regulations.

Samples were collected by the Vacutainer technique into one plain tube used to separate serum for enzyme-linked immunosorbent assay (ELISA) for oxytocin. All samples were collected between 11:00 and 15:00. Samples were centrifuged at $4000 \mathrm{rpm}$ for 7-10 minutes. Serum from plain tubes was transferred into 2 clearly labeled Eppendorf tubes. All aliquots were stored in a deep freezer at $-80^{\circ} \mathrm{C}$.

2.3. Enzyme-Linked Immunosorbent Assay (ELISA). Preparation of reagents, standards, diluents, and buffers took place before starting the ELISA procedure exactly as mentioned in the kits' manual. The competitive ELISA technique was 


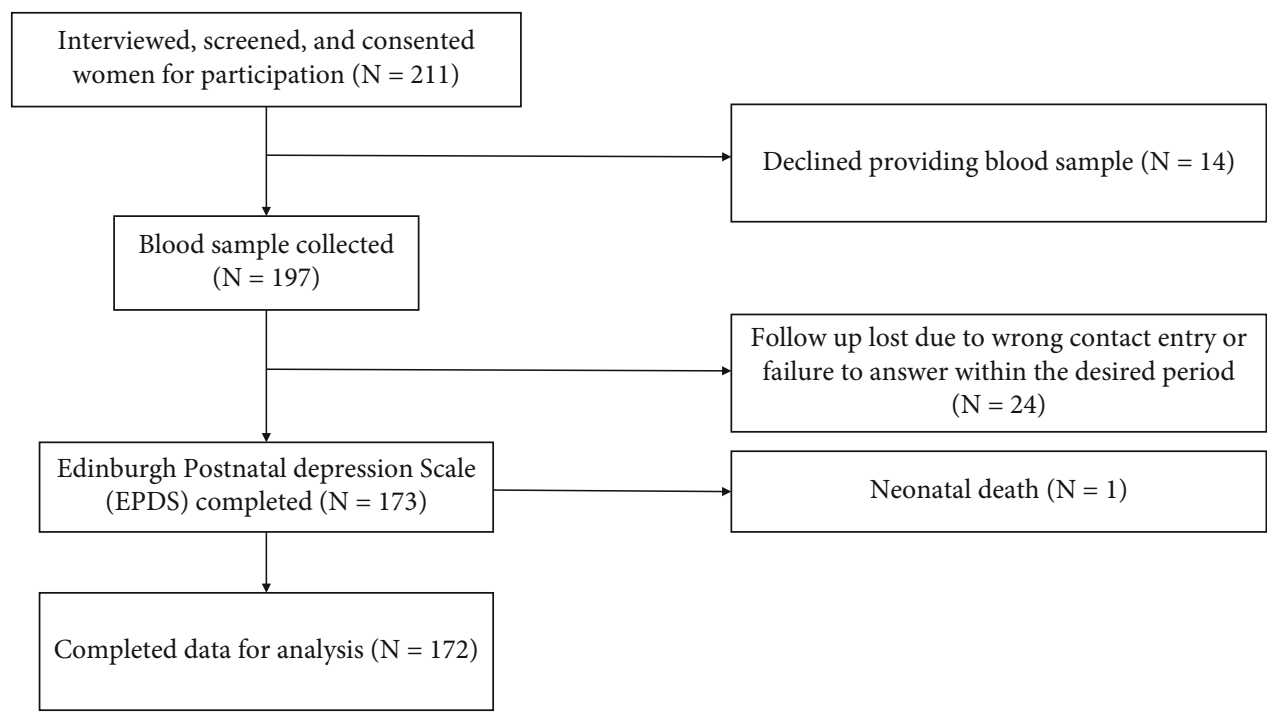

FIgURE 1: Flowchart of study participants.

conducted using kits from Ray Biotech company (Georgia, USA). Series of seven consecutive steps were done using the fully automated ELISA device "Elisys Uno" by HUMAN company (Wiesbaden, Germany).

First, microwell strips were prepared at room temperature. Second, assay diluent B, antioxytocin antibody, standards, and samples were prepared. After that, the antioxytocin antibody was added to all wells and incubated for 1.5 hours at room temperature. Next, the wells were washed with washing buffer four times to remove excess amount of antibodies followed by adding standards and samples to their assigned positions and incubated for 2.5 hours at room temperature. The fifth step included washing the wells again with washing buffer four times to remove excess amount of standards and samples; then, Streptavidin-HRP was added and incubated for 45 minutes at room temperature. Then, all wells were washed with washing buffer four times to remove excess amount of Streptavidin-HRP, and TMB substrate solution was added and incubated for 30 minutes at room temperature avoiding exposure to direct light. Finally, a stop solution was added to all wells and read immediately on a microwell ELISA reader spectrophotometer using $450 \mathrm{~nm}$ as the primary wavelength, before absorbance readings underwent a series of mathematical equations to generate a standard curve and calculate sample results.

2.4. Statistical Analysis. Data were analyzed using the Statistical Package for Social Science (SPSS) version 23 (SPSS, Inc, Chicago, IL). Descriptive statistics including mean $(M)$, standard deviation (SD), and frequency (\%) were utilized to describe the sample. The independent sample $t$-test was used to examine differences in depression scores based on selected demographic, psychosocial, and clinical variables. Pearson product-moment correlation was used to assess the relationship between EPDS and age, maternal age, and oxytocin serum concentration. The level of significance for all analyses was set at $\alpha=0.05$.

\section{Results}

3.1. Sample Characteristics. The mean age for the participants was $31(\mathrm{SD}=4.96)$ ranging from 18 to 41 years old. The majority of participants were well educated as more than three-quarters of them had a bachelor's degree or above $(n=134)$. Although half of the participants were employed $(n=86)$, most of them had a total family monthly income of less than 1000 Jordanian dinars $(\approx 1400$ US dollars) $(n=128)$. More than half of the participants gave birth to a newborn of the female gender $(n=91)$.

The EPDS was normally distributed where the skewness level was 0.1 . Fifty-three participants have scored 13 or above at the EPDS revealing a prevalence of PPD among our cohort to be $30.8 \%$. The mean score of participants at EPDS was $9.98(\mathrm{SD}=5.13)$, ranging from 0 to 24 .

About a quarter $(26 \%, n=45)$ had a previous history of depression, while only $4 \%$ had a family history of depression $(n=7)$. The majority of participants $(90 \%, n=155)$ felt that they had adequate family support, and another $90 \%$ were satisfied by the health care services received during their stay in the hospital. Only $3.5 \%$ of the sample had family issues $(n=6)$.

Seventy-six percent of the pregnancies were planned $(n=131)$. Almost one-fifth of the sample were primgravid $(n=34)$. The mean gestational age of delivery was 37.8 (SD: 1.31 ) ranging from 35 to 41 weeks. About half of the deliveries were elective caesarean section $(n=85), 12.7 \%$ were emergency caesarean section $(n=22)$, and $37 \%$ were vaginal deliveries $(n=64)$. Postpartum haemorrhage complicated six deliveries.

Among the 64 participants who delivered vaginally, 79\% did not receive any medication for pain management. Episiotomy and repair of perineal tears were performed for $15 \%$ $(n=26)$ and $26 \%(n=44)$ of the sample, respectively.

The mean serum oxytocin concentration was $195.10 \mathrm{ng} /$ $\mathrm{ml}(\mathrm{SD}=123.62)$ ranging from 10.38 to 498.78 . The mean concentration for participants who scored less than 13 was 
TABle 1: Sample characteristics for the participants. Mean: $M$; standard deviation: SD; \%: percent $(N=172)$.

\begin{tabular}{|c|c|}
\hline Variable & $N(\%)$ \\
\hline \multicolumn{2}{|l|}{ Demographic variables } \\
\hline Age & $172(100)$ \\
\hline \multicolumn{2}{|l|}{ Employment } \\
\hline Employed & $86(50)$ \\
\hline Housewife & $86(50)$ \\
\hline \multicolumn{2}{|l|}{ Income } \\
\hline Low & $128(74.70)$ \\
\hline High & $044(25.60)$ \\
\hline \multicolumn{2}{|l|}{ Gender of the baby } \\
\hline Male & $81(47.10)$ \\
\hline Female & $91(52.90)$ \\
\hline \multicolumn{2}{|l|}{ Level of education } \\
\hline School & $25(14.50)$ \\
\hline Diploma & $13(07.60)$ \\
\hline Bachelor & $121(70.30)$ \\
\hline Master & $11(06.40)$ \\
\hline Philosophy degree & $2(01.20)$ \\
\hline \multicolumn{2}{|l|}{ Psychosocial variables } \\
\hline Edinburgh Postnatal Depression Scale (EPDS) & $172(100)$ \\
\hline \multicolumn{2}{|l|}{ EPDS score } \\
\hline$<13$ & $119(69.20)$ \\
\hline$\geq 13$ & $053(30.80)$ \\
\hline \multicolumn{2}{|l|}{ Previous history of depression } \\
\hline Yes & $045(26.20)$ \\
\hline No & $127(73.80)$ \\
\hline \multicolumn{2}{|l|}{ Family history of depression } \\
\hline Yes & $007(04.10)$ \\
\hline No & $165(95.90)$ \\
\hline \multicolumn{2}{|l|}{ Family emotional support } \\
\hline Yes & $155(90.10)$ \\
\hline No & $017(09.90)$ \\
\hline \multicolumn{2}{|l|}{ Family issues } \\
\hline Yes & $006(03.50)$ \\
\hline No & $166(96.50)$ \\
\hline \multicolumn{2}{|l|}{ Satisfied with health care } \\
\hline Yes & $155(90.10)$ \\
\hline No & $007(09.90)$ \\
\hline \multicolumn{2}{|l|}{ Clinical variables } \\
\hline Serum oxytocin concentration $(\mathrm{ng} / \mathrm{ml})$ & $172(100)$ \\
\hline \multicolumn{2}{|l|}{ Gestational age (weeks) } \\
\hline \multicolumn{2}{|l|}{ Planned pregnancy } \\
\hline Yes & $131(76.20)$ \\
\hline No & $041(23.80)$ \\
\hline \multicolumn{2}{|l|}{ Parity } \\
\hline Prim gravid & $034(19.80)$ \\
\hline Multigravida & $138(80.20)$ \\
\hline
\end{tabular}

TABLe 1: Continued.

\begin{tabular}{|c|c|}
\hline Variable & $N(\%)$ \\
\hline \multicolumn{2}{|c|}{ Presence history of miscarriages } \\
\hline Yes & $047(27.30)$ \\
\hline No & $125(72.70)$ \\
\hline \multicolumn{2}{|l|}{ Mode of delivery } \\
\hline Caesarean section & $108(62.80)$ \\
\hline Vaginal delivery & $064(37.20)$ \\
\hline \multicolumn{2}{|c|}{ Analgesics use during labour } \\
\hline Yes & $036(56.25)$ \\
\hline No & $028(43.75)$ \\
\hline \multicolumn{2}{|l|}{ Episiotomy } \\
\hline Yes & $026(40.62)$ \\
\hline No & $038(59.38)$ \\
\hline \multicolumn{2}{|l|}{ Perineal suturing } \\
\hline Yes & $044(68.75)$ \\
\hline No & $020(31.25)$ \\
\hline \multicolumn{2}{|c|}{ Postpartum haemorrhage } \\
\hline Yes & $006(03.50)$ \\
\hline No & $166(96.50)$ \\
\hline \multicolumn{2}{|l|}{ Gestational diabetes } \\
\hline Yes & $002(01.20)$ \\
\hline No & $170(98.80)$ \\
\hline \multicolumn{2}{|l|}{ Induced labour } \\
\hline Yes & $013(07.60)$ \\
\hline No & $159(92.40)$ \\
\hline
\end{tabular}

184.16, while it was 219 for participants who scored 13 or more. The difference between the means of oxytocin concentration for both groups was not significant, as the $t$-test illustrated.

Results of the sample characteristics are presented in Table 1.

No missing or outlier values were detected in the analyzed data.

3.2. EPDS Scores and Serum Oxytocin Concentration. Pearson $r$ indicated that there was a nonsignificant direct correlation between EPDS scores and serum oxytocin concentration. On the other hand, there was a nonsignificant inverse correlation $r(43)=-0.13, p=0.37$ between the EPDS scores and the serum oxytocin concentration among women with a lifetime history of depression, as shown in Table 2.

3.3. EPDS Scores and Selected Psychosocial and Clinical Variables. As illustrated in Table 3, the independent $t$-test has shown that there was a statistically significant difference $t(170)=-4.40, p<0.001$, between the mean EPDS score of participants with a previous lifetime history of depression $(M=12.73, \mathrm{SD}=4.76)$ and EPDS score of participants without previous history of depression $(M=9.01, \mathrm{SD}=4.91)$. Also, there was a statistically significant association between the mean EPDS score and the level of education, level of 
TABle 2: Pearson product-moment correlation to assess the correlation between Edinburgh Postnatal Depression Scale (EPDS) scores with maternal age, serum oxytocin concentration, and gestational age of delivery $(N=172)$.

\begin{tabular}{lcc}
\hline Variable & EPDS scores $r$ & $p$ value \\
\hline Maternal age & 0.02 & 0.76 \\
Oxytocin serum concentration & 0.10 & 0.23 \\
Gestational age of delivery & 0.05 & 0.54 \\
\hline
\end{tabular}

income, family history of depression, family issues, and emotional family support.

Participants with low education, low income, previous history of depression, positive family history of depression, positive family issues, and absent emotional family support have scored significantly higher on EPDS score than their counterparts.

Table 2 demonstrates that there was no relationship between EPDS scores with the gestational age of delivery nor maternal age using Pearson $r$ test.

\section{Discussion}

Postpartum depression (PPD) continues to represent a major public health problem. Many studies were carried out to define the possible etiologic factors behind it. Some have suggested psychosocial factors like poverty, low social support, pain, stress, and anxiety to play a major role [19], while other studies have focused more on the biochemical background suggesting neuroendocrinological factors like Estradiol and oxytocin to be causative factors [12, 20]. The rest of the studies have highlighted the role of the genetic predisposition and the family history of depression [21].

Due to the available evidence showing the link between oxytocin with maternal behavior, mother-infant bonding, and stress-related disorders along with its antidepressant and anxiolytic properties, we have established this study primarily to test the association between oxytocin and PPD $[14,22]$. At the same time, we have studied the associations between PPD and other psychosocial and clinical variables as potential etiologic factors suggested in the literature [19].

Unlike our proposed assumption, we found no significant association between antenatal serum oxytocin concentration in the late third-trimester (34 to 36 weeks) and early postpartum depression symptoms (4 to 6 weeks). Hence, isolated late trimester oxytocin concentration is not a useful marker for early-onset PPD. On the other hand, there was a statistically significant association between PPD and below bachelor's degree level of education, below 1000 Jordanian dinars $(\approx 1400$ US dollars) family monthly income, positive family history of depression, positive family issues, previous history of depression, and absent emotional family support. Therefore, it is recommended to include these variables during screening process for PPD, with special focus on the presence of a previous history of depression.

Regarding the association with oxytocin, our results partially agreed with Massey et al. who at first found no
TABLE 3: Independent $t$-test to examine the difference in Edinburgh Postnatal Depression Scale (EPDS) based on selected demographic, social and psychological, and clinical variables $(N=172)$.

\begin{tabular}{|c|c|c|c|}
\hline Variable & $M(\mathrm{SD})$ & $t(170)$ & $p$ value \\
\hline Employment & & 1.20 & 0.23 \\
\hline Employed & $09.51(5.09)$ & & \\
\hline Nonemployed & $10.45(5.15)$ & & \\
\hline Income & & 2.17 & 0.03 \\
\hline Low & $10.48(4.95)$ & & \\
\hline High & $08.55(5.42)$ & & \\
\hline Gender of the newborn & & 1.08 & 0.27 \\
\hline Male & $09.53(4.78)$ & & \\
\hline Female & $10.38(5.41)$ & & \\
\hline Education & & 2.50 & 0.01 \\
\hline Below bachelor & $12.32(5.46)$ & & \\
\hline Bachelor and above & $09.59(4.98)$ & & \\
\hline Previous history of depression & & -4.40 & $<0.001$ \\
\hline Yes & $12.73(4.76)$ & & \\
\hline No & $09.01(4.91)$ & & \\
\hline Family history of depression & & -2.45 & 0.01 \\
\hline Yes & $14.57(5.25)$ & & \\
\hline No & $9.79(5.05)$ & & \\
\hline \multicolumn{4}{|l|}{ Family emotional support } \\
\hline Yes & $9.71(5.02)$ & 2.12 & 0.03 \\
\hline No & $12.47(5.60)$ & & \\
\hline \multicolumn{4}{|l|}{ Family issues } \\
\hline Yes & $15.50(5.71)$ & -2.73 & 0.007 \\
\hline No & $9.78(5.01)$ & & \\
\hline Satisfied with health care & & -0.73 & 0.46 \\
\hline Yes & $10.08(5.12)$ & & \\
\hline No & $09.12(5.27)$ & & \\
\hline Planned pregnancy & & 1.49 & 0.13 \\
\hline Yes & $09.66(5.01)$ & & \\
\hline No & $11.02(5.43)$ & & \\
\hline Parity & & 1.81 & 0.07 \\
\hline Primgravid & $08.56(4.56)$ & & \\
\hline Multigravida & $10.33(5.22)$ & & \\
\hline Previous history of miscarriages & & -0.46 & 0.64 \\
\hline Yes & $10.28(5.05)$ & & \\
\hline No & $09.87(5.17)$ & & \\
\hline Mode of delivery & & -1.04 & 0.29 \\
\hline Caesarean section & $10.30(5.36)$ & & \\
\hline Vaginal delivery & $9.45(4.70)$ & & \\
\hline Postpartum haemorrhage & & -0.65 & 0.51 \\
\hline Yes & $11.33(5.27)$ & & \\
\hline No & $09.93(5.13)$ & & \\
\hline Induced labour & & 0.99 & 0.31 \\
\hline Yes & $08.62(5.47)$ & & \\
\hline No & $10.09(5.10)$ & & \\
\hline
\end{tabular}


correlation between antenatal oxytocin and PPD symptoms. But, a direct significant association appeared only among women with a lifetime history of depression [23]. In fact, when we tested oxytocin with postpartum depression among women with a lifetime history of depression, similar to [23], we found a nonsignificant inverse correlation.

In comparison with [12], our results were not similar. Their study showed a significant inverse correlation between antenatal oxytocin and PPD symptoms. They demonstrated that plasma oxytocin level significantly predicted PPD symptoms 2 weeks after delivery. Also, they found a significant correlation between oxytocin level and gestational age at delivery [12]. Although the settings of the study carried out by [12] were very similar, we did not find the inverse correlation between oxytocin and PPD symptoms, nor we found any relation between oxytocin and gestational age at delivery. This variance might be attributed to the difference in the number of participants $(74: 172)$, the difference in the ethnic groups of participants (Switzerland:Jordan), the difference of the cut-point levels of EPDS (10:13), and the difference in the time of assessment of PPD symptoms (2 weeks : 4 to 6 weeks).

In comparison with Eapen et al., they ended up finding no correlation between antenatal oxytocin and postpartum depression, similar to our results. However, a significant inverse correlation was drawn between serum oxytocin 3 months after delivery with antenatal and postnatal depression [24].

Indeed, the available results from our work and the aforementioned works have generated a more complicated picture of the association between antenatal oxytocin and PPD. This conclusion was previously suggested by two recent systematic reviews by Moura et al. and Thul et al. testing the association between antenatal and postnatal oxytocin concentration with PPD symptoms, where they have found inconclusive results [25, 26]. Therefore, we are proposing some possible available theories that might be able to explain the disintegration between peripheral oxytocin level and its effect centrally. First, Cyranowski et al. suggested that peripheral oxytocin may have a dysregulated pattern of release in depressed women making it unreliable [27]. Second, the genetic variation in the oxytocin receptor gene may play a role in modulating the response of different candidates to the same stimuli, as suggested by Chen et al. and Jonas et al. [28, 29]. Third, there may be different signals that alter the oxytocin physiological response. This theory was suggested by Stubue et al. after they found that breastfeeding had intensified the relationship between oxytocin and PPD [30].

Regarding the association of PPD with psychosocial factors, in comparison between our study and a previous study that was conducted in the north of Jordan, as well, we ended up with similar results in terms of the significant association between PPD with low income and poor family emotional support.

Our study has highlighted the alarming prevalence of PPD among Jordanian mothers sitting at 30.8\%. Furthermore, 13 participants (out of 172 , representing $7.5 \%$ ) have admitted that they had thoughts of harming themselves at different frequencies. One of them had these thoughts quite often.

4.1. Limitations. Although this study is the first-of-its-kind that tested the association between PPD and antenatal serum oxytocin concentration among Middle Eastern women, and the study sample used was the biggest, there were some limitations in the study. The first limitation is that the sample was collected from a single hospital where most women descended from the same ethnic, social, and environmental background, which makes it difficult to generalize the findings on all women. The second limitation is that EPDS has limited sensitivity and specificity [31]. Future researchers are encouraged to combine oxytocin levels with other biological, psychosocial, or clinical variables before testing its association with postpartum depression. The third limitation is that we have not assessed the change in oxytocin level (postpartum level compared with antenatal level) which is more important than a single antenatal value. Future studies are recommended to assess the association between oxytocin transition and PPD.

\section{Conclusions}

About one in three women in Jordan suffers from postpartum depression. Therefore, active interventions should be implemented immediately including antenatal and postnatal screening for PPD.

There was no association between late antenatal thirdtrimester serum oxytocin level and early-onset postpartum depression symptoms. A significant association was found between PPD and low educational level, low income, positive family history of depression, positive family issues, previous history of depression, and absent emotional family support, making these variables essential during screening for PPD. The association between PPD and previous lifetime history of depression was noticeable.

\section{Data Availability}

The data that support the findings of this study are available from King Abdulla University Hospital. Restrictions apply to the availability of these data, which were used under license for this study. Data are available from Hasan Rawashdeh with the permission of the IRB committee at King Abdulla University Hospital.

\section{Ethical Approval}

Ethical approval for this study was granted by the IRB committee (Reference number: $48 / 116 / 2016$ on $31^{\text {st }}$ of May 2018). The work was carried out in accordance with The Code of Ethics of the World Medical Association (Declaration of Helsinki).

\section{Consent}

All participants had completed and signed an informed consent for participation. Consents were kept safe and ready to be provided upon request. 


\section{Disclosure}

This paper was posted as a preprint on Research Square on the $8^{\text {th }}$ of Feb 2021 under the title "The Role of Third Trimester Oxytocin Level in Predicting Postpartum Depression Symptoms Among Jordanian Mothers"[32]. The sponsor had no role in collection and interpretation of data, in the writing of the report, and in the decision to submit the article for publication. We acknowledge that the manuscript represents original work and has been submitted solely to the Depression Research and Treatment Journal.

\section{Conflicts of Interest}

The authors declare that they have no competing interests.

\section{Authors' Contributions}

All authors give the publisher the permission to publish the work.

\section{Acknowledgments}

This work was funded by the Deanship of Research at Jordan University of Science and Technology under project grant number (20180434). Their role was limited to laboratory analysis expenses. We are very thankful to the staff at King Abdulla University Hospital for their cooperation during conducting the work, especially nurses at the antenatal clinics and technicians at the laboratories.

\section{References}

[1] J. Dayan, C. Creveuil, M. N. Marks et al., "Prenatal depression, prenatal anxiety, and spontaneous preterm birth: a prospective cohort study among women with early and regular care," Psychosomatic Medicine, vol. 68, no. 6, pp. 938-946, 2006.

[2] A. Gmitrowicz and A. Kucharska, "Developmental disorders in the fourth edition of the American classification: diagnostic and statistical manual of mental disorders (DSM IV - optional book)," Psychiatria Polska, vol. 28, no. 5, pp. 509-521, 1994.

[3] M. Chaaya, O. M. Campbell, F. El Kak, D. Shaar, H. Harb, and A. Kaddour, "Postpartum depression: prevalence and determinants in Lebanon," Archives of Women's Mental Health, vol. 5, no. 2, pp. 65-72, 2002.

[4] K. I. Mohammad, J. Gamble, and D. K. Creedy, "Prevalence and factors associated with the development of antenatal and postnatal depression among Jordanian women," Midwifery, vol. 27, no. 6, pp. e238-e245, 2011.

[5] N. K. Ozcan, N. E. Boyacioglu, and H. Dinc, "Postpartum depression prevalence and risk factors in Turkey: a systematic review and meta-analysis," Archives of Psychiatric Nursing, vol. 31, no. 4, pp. 420-428, 2017.

[6] N. L. Davis, A. N. Smoots, and D. A. Goodman, "Pregnancy-related deaths: data from 14 US maternal mortality review committees, 2008-2017," Education, vol. 40, no. 36, 2019.

[7] B. L. Bauman, J. Y. Ko, S. Cox et al., "Vital signs: postpartum depressive symptoms and provider discussions about perinatal depression-United States, 2018," Morbidity and Mortality Weekly Report, vol. 69, no. 19, pp. 575-581, 2020.
[8] K. L. Wisner, J. M. Perel, K. S. Peindl, B. H. Hanusa, C. M. Piontek, and R. L. Findling, "Prevention of postpartum depression: a pilot randomized clinical trial," The American Journal of Psychiatry, vol. 161, no. 7, pp. 12901292, 2004.

[9] C. Chen, J. Gao, J. Zhang, L. Jia, T. Yu, and Y. Zheng, "Serum leptin level measured $48 \mathrm{~h}$ after delivery is associated with development of postpartum depressive symptoms: a 3-month follow-up study," Archives of Women's Mental Health, vol. 19, no. 6, pp. 1001-1008, 2016.

[10] J. Fung, B. Gelaye, Q. Y. Zhong et al., "Association of decreased serum brain-derived neurotrophic factor (BDNF) concentrations in early pregnancy with antepartum depression," BMC Psychiatry, vol. 15, no. 1, p. 43, 2015.

[11] R. Ramachandran Pillai, L. Sharon, N. R. Premkumar, S. Kattimani, H. Sagili, and S. Rajendiran, "Luteinizing hormone-follicle stimulating hormone ratio as biological predictor of post-partum depression," Comprehensive Psychiatry, vol. 72, pp. 25-33, 2017.

[12] M. Skrundz, M. Bolten, I. Nast, D. H. Hellhammer, and G. Meinlschmidt, "Plasma oxytocin concentration during pregnancy is associated with development of postpartum depression," Neuropsychopharmacology, vol. 36, no. 9, pp. 1886-1893, 2011.

[13] K. Macdonald and T. M. Macdonald, "The peptide that binds: a systematic review of oxytocin and its prosocial effects in humans," Harvard Review of Psychiatry, vol. 18, no. 1, pp. 121, 2010.

[14] I. D. Neumann and R. Landgraf, "Balance of brain oxytocin and vasopressin: implications for anxiety, depression, and social behaviors," Trends in Neurosciences, vol. 35, no. 11, pp. 649-659, 2012.

[15] F. Faul, E. Erdfelder, A.-G. Lang, and A. Buchner, "G* Power 3: a flexible statistical power analysis program for the social, behavioral, and biomedical sciences," Behavior Research Methods, vol. 39, no. 2, pp. 175-191, 2007.

[16] J. L. Cox, J. M. Holden, and R. Sagovsky, "Detection of postnatal depression. Development of the 10-item Edinburgh Postnatal Depression Scale," The British Journal of Psychiatry, vol. 150, no. 6, pp. 782-786, 1987.

[17] R. Ghubash, M. T. Abou-Saleh, and T. K. Daradkeh, "The validity of the Arabic Edinburgh Postnatal Depression Scale," Social Psychiatry and Psychiatric Epidemiology, vol. 32, no. 8, pp. 474-476, 1997.

[18] J. Evans, J. Heron, H. Francomb, S. Oke, and J. Golding, "Cohort study of depressed mood during pregnancy and after childbirth," BMJ, vol. 323, no. 7307, pp. 257-260, 2001.

[19] C. T. Beck, "Predictors of postpartum depression: an update," Nursing Research, vol. 50, no. 5, pp. 275-285, 2001.

[20] J. L. Workman, C. K. Barha, and L. A. M. Galea, "Endocrine substrates of cognitive and affective changes during pregnancy and postpartum," Behavioral Neuroscience, vol. 126, no. 1, pp. 54-72, 2012.

[21] B. Doornbos, D. A. J. Dijck-Brouwer, I. P. Kema et al., "The development of peripartum depressive symptoms is associated with gene polymorphisms of MAOA, 5-HTT and COMT," Progress in Neuro-Psychopharmacology and Biological Psychiatry, vol. 33, no. 7, pp. 1250-1254, 2009.

[22] R. Feldman, A. Weller, O. Zagoory-Sharon, and A. Levine, "Evidence for a neuroendocrinological foundation of human affiliation: plasma oxytocin levels across pregnancy and the 
postpartum period predict mother-infant bonding," Psychological Science, vol. 18, no. 11, pp. 965-970, 2007.

[23] S. H. Massey, S. A. Schuette, H. Pournajafi-Nazarloo, K. L. Wisner, and C. S. Carter, "Interaction of oxytocin level and past depression may predict postpartum depressive symptom severity," Archives of Women's Mental Health, vol. 19, no. 5, pp. 799-808, 2016.

[24] V. Eapen, M. Dadds, B. Barnett et al., "Separation anxiety, attachment and inter-personal representations: disentangling the role of oxytocin in the perinatal period," PLoS One, vol. 9, no. 9, article e107745, 2014.

[25] D. Moura, M. C. Canavarro, and M. Figueiredo-Braga, "Oxytocin and depression in the perinatal period-a systematic review," Archives of Women's Mental Health, vol. 19, no. 4, pp. 561-570, 2016.

[26] T. A. Thul, E. J. Corwin, N. S. Carlson, P. A. Brennan, and L. J. Young, "Oxytocin and postpartum depression: a systematic review," Psychoneuroendocrinology, vol. 120, article 104793, 2020.

[27] J. M. Cyranowski, T. L. Hofkens, E. Frank, H. Seltman, H. M. Cai, and J. A. Amico, "Evidence of dysregulated peripheral oxytocin release among depressed women," Psychosomatic Medicine, vol. 70, no. 9, pp. 967-975, 2008.

[28] F. S. Chen, R. Kumsta, B. von Dawans, M. Monakhov, R. P. Ebstein, and M. Heinrichs, "Common oxytocin receptor gene (OXTR) polymorphism and social support interact to reduce stress in humans," Proceedings of the National Academy of Sciences of the United States of America, vol. 108, no. 50, pp. 19937-19942, 2011.

[29] W. Jonas, V. Mileva-Seitz, A. W. Girard et al., "Genetic variation in oxytocin rs2740210 and early adversity associated with postpartum depression and breastfeeding duration," Genes, Brain, and Behavior, vol. 12, no. 7, pp. 681-694, 2013.

[30] A. M. Stuebe, K. Grewen, and S. Meltzer-Brody, "Association between maternal mood and oxytocin response to breastfeeding," Journal of Women's Health, vol. 22, no. 4, pp. 352-361, 2013.

[31] J. Milgrom, J. Mendelsohn, and A. W. Gemmill, "Does postnatal depression screening work? Throwing out the bathwater, keeping the baby," Journal of Affective Disorders, vol. 132, no. 3, pp. 301-310, 2011.

[32] H. Rawashdeh, Z. Alalwani, A. Sindiani, R. Alodetalah, and M. Alqudah, The role of third trimester oxytocin level in predicting postpartum depression symptoms among Jordanian mothers, Research Square, 2021. 